
\title{
CResearch Square \\ The Risk Assessment of Thyroid Disorders Based on Metabonomics
}

\section{Jing Bai}

Beijing Tongren Hospital Department of Clinical Labortory

Xiangyi Liu ( $\sim$ baijingguoduo@163.com )

Beijing Tongren Hospital Department of Clinical Laboratory https://orcid.org/0000-0001-8840-5181

Haishun Liu

Capital Normal University Department of Physics

Chan Xiong

Institute of Chemistry,NAWI Graz,University of Graz

Kewen Zhu

Bioyong Technology Co.Ltd.,Beijing

Qingwei Ma

Bioyong Technology Co.Ltd.,Beijing

\section{Research article}

Keywords: Thyroid disease, Thyroid hormones, Trace elements, Random forest model

Posted Date: March 2nd, 2021

DOI: https://doi.org/10.21203/rs.3.rs-279303/v1

License: (c) (i) This work is licensed under a Creative Commons Attribution 4.0 International License.

Read Full License 


\section{Abstract}

\section{Background}

When thyroid disorders occurred, it can disturb the balance of trace elements in human body. Therefore, the detection of trace elements during an early screening of thyroid diseases, treatment, and prognosis judgment based on personal characteristics of drugs and treatment will provide a scientific basis for clinical decision-making and can effectively reduce patients' pain and social medical burden.

\section{Methods}

50 healthy volunteers and 200 patients with thyroid diseases (58 cases of thyroid nodule, 59 cases of goiter, 34 cases of postoperative thyroid cancer and 24 cases of thyroid cancer) were compared in our study. Total trace elements of iodine, selenium, manganese, chromium etc. (in total 28 elements) in human serum were detected by inductively coupled plasma mass spectrometry (ICP-MS). A risk assessment model for thyroid disease was established after analyzing trace elements content, thyroid function and ultrasonography results by gender and age with the characteristics of the Chinese population.

\section{Results}

The results showed there were significant differences in eight trace elements (I, Ca, $\mathrm{Fe}, \mathrm{Ni}, \mathrm{Cu}, \mathrm{Zn}, \mathrm{Se}, \mathrm{Sb})$ between the thyroid disease group and the healthy control group $(P<0.05)$. There were significant differences in ten trace elements (I, $\mathrm{Ca}, \mathrm{Cr}, \mathrm{Mn}, \mathrm{Co}, \mathrm{Ni}, \mathrm{Cu}, \mathrm{Zn}, \mathrm{Se}, \mathrm{Sb})$ between the female thyroid disease group and the control group $(P<0.05)$. And there were significant differences in the eight trace elements $(\mathrm{I}$, $\mathrm{Mn}, \mathrm{Fe}, \mathrm{Ni}, \mathrm{Cu}, \mathrm{Zn}, \mathrm{Se}, \mathrm{Sb})$ between the male thyroid disease group and the control group $(P<0.05)$. There were significant correlations between the contents of FT3, FT4, T3 and T4 corresponding to iodine $(P<$ $0.05)$ in thyroid disease group. Also, correlations between Fe-Zn, Cr-Mn, Ca-Zn, Ca-Se, Ca-Fe and Zn-Se $(P<0.05)$ were found in thyroid disease group too.

\section{Conclusions}

The high concentration of iodine and manganese are risk factors of thyroid disease. In addition to the low concentration of cobalt, nickel, copper, zinc, selenium, and antimony, calcium in the serum of female patients with thyroid disease and iron in the serum of male patients with thyroid disease play a very important role. Random forest model based on element risk assessment of thyroid disease is helpful for the diagnosis and treatment of thyroid disease.

\section{Background}

Thyroid disease has the second-highest incidence rate among endocrine diseases, only after diabetes. Globally, $8 \%-20 \%$ of adults suffer from thyroid disease. In areas where endemic diseases are prevalent, the incidence rate is even higher, which can exceed $50 \%$ [1-2]. Thyroid cancer accounts for $1 \% \sim 3 \%$ of all 
cancers according to previous research[3]. Worldwide, the incidence rate of thyroid disease including thyroid cancer was increasing. In China, thyroid cancer ranks third female solid tumors, and has become the fastest-growing incidence of malignant solid tumors[4].

Because the symptoms of thyroid diseases are hidden, the high-risk group should check the thyroid function according to their symptoms regularly. The current examines include high-resolution ultrasound, hypersensitive thyroid stimulating hormone (TSH), fine needle aspiration biopsy (FNAB), and analysis combined with clinical history and physical examination findings. However, the pathological reports of patients who cannot diagnose the nature of thyroid nodules before operation show that $20 \%$ of the thyroid nodules are malignant and $80 \%$ of the patients receive unnecessary partial or total thyroidectomy [5]. Once the surgery is operated, the life quality of patients will be seriously affected. Unaesthetic and patients need hormone replacement therapy for life after surgery. On the other hand, the medical costs involved are also significantly different [6] $₫$ which seriously consumes medical and social resources, and has become an important public health problem in China.

Trace elements are affected by different mechanisms or thyroid hormones, and are closely related to various thyroid diseases. In 2004, Haraguchi proposed the concept of metallomics, because the research objects of metallomics also include non-metallic trace elements, such as selenium (the component of glutathione peroxidase), arsenic (the role of activating zinc) and iodine (the role of activating thyroid), which can also be called as elemental omics[7-8]. Trace elements play an important role in the metabolism and growth of the human body. Thyroid disease will affect the metabolism balance of trace elements in the body. In turn, the imbalance of trace elements will aggravate the condition of patients with thyroid disease. It is reported that the level of urinary iodine is positively correlated with the incidence rate of thyroid cancer, and there is a significant correlation between high urinary iodine and thyroid malignancy and thyroid cancer metastasis. Selenium level is negatively correlated with thyroid cancer incidence rate. [9-10] At present, there are few reports about the relationship between metabonomics and clinical diseases. This paper studies the relationship between trace elements and thyroid diseases, to provide new ideas for the treatment of thyroid diseases.

With the increasingly clear relationship between trace elements and diseases, accurate, rapid, and convenient determination of trace elements in the human body has become an urgent issue in the field of trace element detection. Therefore, the detection of trace elements, early screening of thyroid diseases, treatment, and prognosis judgment based on personal characteristics of drugs and treatment, to provide a scientific basis for clinical decision-making, can effectively reduce personal pain and social medical burden.

On the basis of detecting the element group of normal people and patients with thyroid disease, this paper attempts to establish a Random forest model for risk assessment of thyroid nodule with iodine, selenium, calcium, iron, zinc and other trace elements, providing scientific and effective data support for clinical diagnosis and treatment of thyroid nodule, to promote standardized diagnosis and treatment of thyroid nodule in China. Meanwhile, avoiding benign nodule operation can improve the early detection 
rate of thyroid cancer, reduce the death risk of thyroid cancer, and improve the survival rate and life quality.

\section{Methods}

\section{Subjects}

This study was conducted in the Department of Medical Laboratory, Tongren Hospital from January 2019 to December 2019. It was performed following the guidelines of the Declaration of Helsinki Principles and approved by the Institutional Review Board of Tongren Hospital (approval number 2019-0907). All subjects gave written informed consent. 50 healthy people with physical examination were randomly selected as the control group from January to December 2019. Above mentioned 50 healthy people who received a comprehensive examination in the physical examination center of our hospital were healthy people without an adenoid disease (normal thyroid).

The enrollment criteria were: 200 cases of thyroid operation in Tongren Hospital, Capital Medical University, older than 18 years old with pathological results confirmed thyroid cancer, thyroid nodule diameter is not less than $1 \mathrm{~cm}$. Among them, 58 cases of thyroid nodule, 59 cases of goiter, 34 cases of postoperative thyroid, and 24 cases of thyroid cancer, other types of patients cannot be statistically analyzed due to the small sample size (less than 3 cases). Exclude Standard: thyroid function test and ultrasonography results were not from our hospital (ensure the measurement method and level of data sources are consistent); combination a serious chronic disease, such as kidney failure, liver disease, or heart disease; cancer; recurrent TC; thyroid autoimmune disease; surgical history within 6 months before the study; smoking; take thyroid related drugs before testing; pregnantor lactating women.

\section{Sampling}

$10 \mathrm{~mL}$ venous blood was collected, coagulated for $30 \mathrm{~min}$, then centrifuged for 10 min $\left(16,000 \mathrm{~g}, 4^{\circ} \mathrm{C}\right)$. Upper phase of serum was taken and stored in $2 \mathrm{~mL}$ Eppendorf tubes at $-80^{\circ} \mathrm{C}$ before analysis.

\section{Analysis}

Thyroid volume was measured by using GE LOGIQ 9 and Philips iu22 colors Doppler ultrasound diagnostic instrument and linear array probe (frequency 5-14 MHz). The concentrations of serum FT3, FT4, T3, T4, TSH, TgAbs, TPOAb, and TSH were measured by Chemiluminescent immunoassay analysis using reagent pack (Roche Germany). The following are reference ranges: 3.1-6.8 pmol/L for FT3, 12-22 $\mathrm{pmol} / \mathrm{L}$ for FT4, 1.3-3.1 nmol/L for T3, 66-181 nmol/L for T4, 0.27-4.2 mIU/L for TSH, <115 IU/mL for TgAbs, and 0-34 IU/mL for TPOAb. ICPMS (Clin-ICP-QMS-I, Bioyong Technology Co., Ltd., Beijing, China) was used for trace element analysis. The content of trace elements in the serum is very low $(\mathrm{ng} / \mathrm{L} \sim \mu \mathrm{g} / \mathrm{L})$ and there are several uncertain factors in the detection process caused by the complex matrix of human serum. The collision reaction cell is helpful to reduce interference and increase the detection limit of 
elements. Therefore, we use He mode to detect all 28 elements in this test. The basic parameter settings of the instrument are shown in Table 1.

Ultrapure water used in the experiment was prepared by Milli Q ultrapure water meter (Millipore, Austria), Nitric acid (Rotipuran ${ }^{\circledR} \geq 65 \%$, P.A., ISO, Roth, Karlsruhe, Germany) purified by low boiling point distillation. The single standard solution used in the experiment was calcium, iron, zinc, iodine, lithium, vanadium, chromium, manganese, cobalt, nickel, copper, gallium, arsenic, selenium, strontium, strontium, molybdenum, palladium, cadmium, tin, antimony, tellurium, barium, cerium, platinum, mercury, thallium, lead, bismuth, germanium, germanium, indium, terbium, and rhodium. (1000 $\mu \mathrm{g} / \mathrm{ml}$, National Center for Analysis and Testing of Non-Ferrous Metals and Electronic Materials, China)

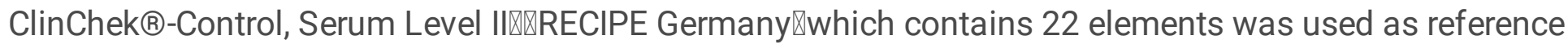
material. Dilution of multi-element test sample: $200 \mu \mathrm{l}$ serum was diluted with $2 \%$ (V/V) nitric acid 1:10, then vibrated for 30 seconds. Dilution of iodine test sample: $200 \mu \mathrm{l}$ serum diluted with $5 \%(\mathrm{~V} / \mathrm{V})$ ammonia solution 1:10, then vibrated for 30 seconds.

The blank reagent, standard curve, and quality control used in the measurement were prepared as the same matrix. The polypropylene tubes used in the sample preparation process were soaked overnight with $10 \%$ nitric acid and washed with ultrapure water for three times.

The standard curve was prepared by mixing a single standard solution. For the six-point standard curve of serum iodine, $5 \%(\mathrm{~V} / \mathrm{V}$ ) ammonia solution was diluted to a concentration of $60,80,120,160,180$, and $200 \mu \mathrm{g} / \mathrm{L}$; for the internal standard, indium was selected, and the internal standard element was also diluted to a final concentration of $20 \mu \mathrm{g} / \mathrm{L}$ by $5 \%(\mathrm{~V} / \mathrm{V})$ ammonia solution. For the six-point standard curve of serum calcium, $5 \%(\mathrm{~V} / \mathrm{V})$ ammonia solution is used to dilute to $2,5,10,50,100$, and $200 \mu \mathrm{g} / \mathrm{L}$; for the six-point standard curve of other elements in serum, $2 \%(V / V)$ nitric acid solution is used to dilute to $0,40,80,120,160$, and $200 \mu \mathrm{g} / \mathrm{L}$; for the internal standard, germanium, indium, rhodium, and terbium were selected; for the internal standard element, $5 \%(\mathrm{~V} / \mathrm{V})$ nitric acid solution was also used to dilute to the final concentration of $20 \mu \mathrm{g} / \mathrm{L}$.

\section{Statistical Analysis}

The software of Clin-ICP-QMS-I was used for quantitative analysis, and Excel (Microsoft Corp., Redmond, WA) and OriginPro9.0 (Northampton, Ma, USA) were used for data statistical analysis. Python 3.6.4 was used for the random forest model. The experimental data were expressed as median and quartiles due to their nonnormal distribution. The correlation between experimental variables was evaluated using Spearman's rank correlation coefficients. Mann-Whitney U test was implemented for non-parametric variables. Different logistic regression models were used to assess the relative risk of a thyroid nodule. All tests were repeated three times and considered statistically significant at $p<0.05$.

\section{Results}




\subsection{Comparison between healthy people $(n=50)$ and patients with thyroid disease $(n=200)$}

Comparison between healthy people $(n=50)$ and patients with thyroid disease $(n=200)$ are shown in Table 2

In table 2, there were eight elements showed significant difference between thyroid disease and control group. Except iodine, the content of all the other elements are relatively low in the serum of thyroid patients compare to the control group. While the content of molybdenum, cadmium, tin, palladium, and thallium in the healthy people and thyroid disease patients is not significantly different.

\subsection{Comparison between healthy people and patients with thyroid disease by gender}

A comparison of the classification of healthy people and patients with thyroid diseases by gender were shown in Table 3. The results of females were shown in Table 4. The results of males were shown in Table 5.

Significant differences in ten trace elements of iodine, calcium, chromium, manganese, cobalt, nickel, copper, zinc, selenium, and antimony were found between female healthy people and female patients with thyroid disease $(P<0.05)$. The content of iodine, chromium, and manganese were increased, while the content of the rest elements were decreased.

Significant differences in the eight trace elements of iodine, manganese, iron, nickel, copper, zinc, selenium, and antimony were found between the male healthy people and male patients with thyroid diseases $(P<0.05)$. Except iodine and manganese, the content of rest elements were decreased in the patients with thyroid diseases compare to the control group.

\subsection{Correlation between trace elements}

We assessed the correlation between concentrations of essential trace elements in both the thyroid disease group and control group. The correlation coefficient is listed in Table 6, and the confidence ellipse between trace elements in thyroid group were shown in Figure 1.

In the thyroid disease group, there were strong correlations between Fe-Zn, Cr-Mn, Ca-Zn, Ca-Se, Ca-Fe and $\mathrm{Zn}-\mathrm{Se}$. Whereas, in the control group, the strong correlation only found between $\mathrm{Ca}-\mathrm{Zn}$ and $\mathrm{Ca}-\mathrm{Se}$, no such correlations were found between other elements. Compared with the control group, the correlation between $\mathrm{Ca}-\mathrm{Zn}$ and $\mathrm{Ca}-\mathrm{Se}$ in the thyroid disease group were stronger. (Figure 1).

\subsection{Correlation between trace element iodine and thyroid function}

This study finds that iodine in thyroid disease group is correlated with T3, T4, FT3 and FT4, and there is

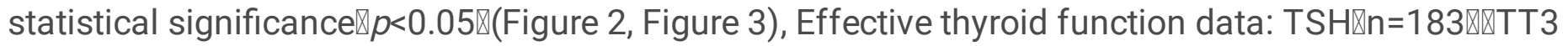

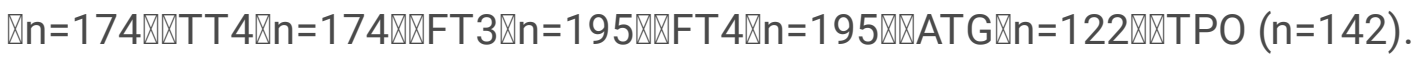

\subsection{Establishment of thyroid disease risk assessment model and its effect evaluation}


Since the accuracy of regression analysis of thyroid diseases (thyroid cancer, thyroid nodule, goiter, and postoperative thyroid surgery) and the classification of healthy people is not very high, we use a random forest algorithm for effective classification.

The values of each index in the data are not all in an order of magnitude. We use the normalization method to preprocess the data. We use a random forest algorithm to model the following four groups of data and divide each group of data into a correction set (70\%) and prediction set (30\%). Because of the randomness of data set partition, each model is run 50 times and the result of prediction set is taken as the final prediction result of each group. From the results in Table 7, we can see that random forest has a good diagnostic effect, with specificity and sensitivity of more than $95 \%$.

\section{Discussion}

China used to be one of the countries with serious iodine deficiency in the world. lodine deficiency may lead to abortion, stillbirth, congenital malformation, endemic cretinism, hypothyroidism, goiter, mental retardation, and other diseases, seriously affecting the quality of the population. [11-12]. Since the implementation of universal salt iodization (USI) in 1995, the research results show that the Median Urinary lodine $\triangle \mathrm{MUI}$ ) of 8 to 10 years old children has increased from $164.8 \mu \mathrm{g} / \mathrm{L}$ in 1995 to $238.6 \mu \mathrm{g} / \mathrm{L}$ in 2011, which exceeds the World Health Organization's best standard for urinary iodine (100-199 $\mathrm{g} / \mathrm{L})$, while it is at the level of iodine excess, 200-299 $\mu \mathrm{g} / \mathrm{L}[13-14]$. Along with the change of iodine nutritional status, the incidence rate of thyroid diseases is changing. It shows that the thyroid disease is developing from simple goiter to toxic nodular goiter, Hashimoto's thyroiditis, and thyroid cancer. [15] Among them, the incidence of thyroid malignancies which is most harmful to human beings annually increase of $14.51 \%,[16]$ and is believed to be caused by excessive iodine intake. However, this conclusion has been controversial [17]. This study shows that a high concentration of iodine is one of the risk factors of thyroid disease. Therefore, we should supplement iodine according to the content of iodine in the serum.

This study also shows that a low concentration of selenium is another of the risk factors for thyroid disease. Selenium has important biological significance for human beings. It is an essential trace element for the human body and has the highest selenium content in the thyroid gland. Selenoprotein is a kind of protein which active center is selenocysteine. At present, 25 kinds of selenoproteins have been found in the human body, mainly including 6 kinds: glutathione peroxidase family, iodothyronine deiodinase family, selenoprotein P, selenoprotein $\mathrm{W}$, thioredoxin reductase and selenophosphate synthetase. Selenium plays a variety of biological functions in the body, such as anti-oxidation, improving immune function, anti-tumor, etc. Selenium is involved in the synthesis, activation, and metabolism of thyroid hormones, and plays an important role in thyroid antioxidant system and immune system [18-20]. Selenium deficiency is closely related to goiter, autoimmune thyroid disease, low T3 syndrome, thyroid cancer, and other diseases [21-22]. Selenium containing preparations can improve thyroid function in some patients with thyroid diseases, which provides a new way for the treatment of thyroid diseases [2325]. A number of studies have shown that in children with goiter in low iodine area, the activities of seleniumand GPx in plasma are significantly reduced. It is concluded that goiter is not only related to 
iodine but plays an important role in low selenium. The mechanism may be as follows: 『low selenium can also cause metabolism disorder of tissue, especially myocardial tissue, dysfunction of mitochondrial oxidative phosphorylation, and the body needs a relatively stable T3 level to ensure that it can provide energy for normal oxidative phosphorylation; deiodinase activity is reduced, T3 production is insufficient, which is not able to meet the needs of the body's metabolism, and TSH rises to feedback to regulate more thyroid secretionT3;囚low selenium reduces the activity of IDI in liver and kidney, decreases the output of T3 in peripheral tissue, and increases the concentration of T4 in blood, decreases the concentration of T3; at the same time, the activity of ID $\otimes$ in pituitary decreases, the output of T3 in pituitary decreases, the

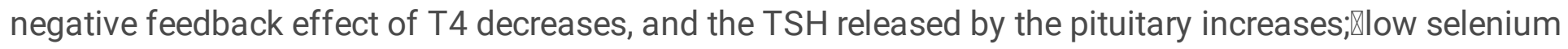
also affected the activity of glutathione peroxidase (GPx) in thyroid, and eliminated $\mathrm{H}_{2} \mathrm{O}_{2}$ produced by cell metabolism The activity of TPO increased, $\mathrm{H}_{2} \mathrm{O}_{2}$ oxidized inorganic iodine to activated iodine, accelerated the synthesis of thyroid hormone, and eliminated $\mathrm{H}_{2} \mathrm{O}_{2}$ at the same time. Due to the above reasons, the synthesis and secretion of thyroid are enhanced, the T3, T4 and iodine reserves in thyroid tissue are insufficient, the iodine intake of thyroid is increased, the protein binding iodine is increased, and the TSH level is increased, resulting in compensatory goiter of thyroid [26-28].

This study shows that manganese plays an important role in thyroid disease. Manganese $(\mathrm{Mn})$ is an essential trace element with potential toxicity under the high level of exposure. As a component of many enzymes and cofactors, manganese plays an important role in many physiological processes in mammals. Manganese superoxide dismutase (Mn SOD) as amanganese-containing enzymeis the main antioxidant enzyme, which can neutralize the toxic effect of active oxygen. Other manganese-containing enzymes include oxidoreductase, transferase, hydrolase, lyase, isomerase, ligase, and glutamine synthetase. Dopamine is also known as an inhibitor of TSH secretion. Dopamine and dopaminergic receptors are involved in neurodevelopment and TSH regulation. Excessive manganese exposure may lead to adverse neurodevelopment results, which is due to the loss of dopaminergic control of TSH regulation of thyroid hormones and the destruction of thyroid homeostasis. This destruction may alter thyroid hormone levels, leading to some defects related to manganese exposure during pregnancy. Dysregulation of thyroid hormone may regulate the production of dopamine through manganese, thus directly or indirectly affecting thyroid function [29].

In females with thyroid disease, calcium plays a very important role. Some studies suggest that females are more likely to have low calcium [30]. Rajinikanth et al. [31] found that the high proportion of parathyroid glands in the thyroid capsule in female patients may be the reason for the high incidence of low calcium. Besides, the gender difference may be related to the effect of sex steroids on the secretion of parathyroid hormone and the genetic variation of the cell signaling pathway. Female menstruation, pregnancy, lactation, and menopause will also cause a lack of calcium and vitamin D. There are much data to show that the lack of vitamin D involves a variety of cancer pathogenesis, including colorectal cancer, prostate cancer, pancreatic cancer, and thyroid cancer. Some experimental studies have proved that vitamin D can induce apoptosis and delay the growth of cancer cells. Clinckspoor et al [32] confirmed the inhibitory effect of 1,25-dihydroxyvitamin D3 on the proliferation of thyroid cancer cells. 
The relationship between vitamin $D$ deficiency and thyroid diseases has become a hot research topic and most research evidence supports the view that vitamin $D$ deficiency can increase the risk of multiple thyroid diseases. However, it is not clear whether vitamin D deficiency has some special mechanism. Furthermore, the vitamin $D$ level of the body is affected by many factors, thus the results of many studies are still objective differences. Regardless of whether vitamin D supplementation has the effect of preventing and treating thyroid diseases, how to supplement vitamin D still needs a lot of research support.

Although there is a significant difference in iron between male patients with thyroid disease and male healthy control group, the incidence of iron deficiency anemia in female patients is relatively high. Many enzymes in the human body need iron as a cofactor, including enzymes involved in the tricarboxylic acid cycle and the electron transfer system. In the absence of iron, the activity of iron-containing enzymes and iron-dependent enzymes in the body is reduced [33], the biochemical reactions catalyzed by these enzymes are blocked, the metabolic function of the body is changed, and the homeostasis of the internal environment is affected. The activation, iodization, and coupling of iodine need the catalysis of TPO. Serious iron deficiency will reduce the activity of TPO, affect the response to iodine in the process of thyroid hormone synthesis, and then affect the synthesis of thyroid hormone [34]. When thyroid function is normal, T3 mainly comes from T4 to T3 transformation of liver and kidney. While, when thyroid function is low, T3 mainly comes from thyroid. The hormone T4 is transformed into T3 with biological activity through the deiodination of the outer ring. This particular pathway is catalyzed by a selenine containing type I deiodinase (ID-I), which is present in high concentrations in the liver, kidney, thyroid, and pituitary. Goswami et al. [35] found that iron ion complex 2,2' dipyridine can strongly inhibit the activity of ID-I, indicating that there may be an iron ion active center to affect the catalytic effect of the enzyme. Therefore, the activity of ID-I decreased and the 5 'deiodination pathway was inhibited in the iron deficiency state, which led to the decrease of T3 in serum. It is also shown that iron deficiency anemia can reduce the response of TSH to Thyrotropin releasing hormone (TRH), and affect the regulation of the central nervous system on thyroid function [36]. Therefore, many countries advocate strengthening iodine nutrition and iron nutrition intake at the same time [37,38], which may be a better control method for IDD in iron deficiency areas.

Random forest is a kind of integrated learning model based on the decision tree, which has a relatively good classification effect.[39,40] It can process high-dimensional feature data, not easy to over fit, and the model training speed is faster. Compared with the random forest algorithm, the logistic regression only evaluates with a single test sample, but its explanation of the influencing factors are vague, and the relative risk of the influencing factors cannot be given and the direction of action. However, the random forest algorithm balances the influence of sample error by a large number of randomly selected samples and classifies and comprehensively evaluate a large number of different test data, which is more reliable than the result of logistic regression fitting.

There are still some deficiencies in this study: the number of cases is not enough and a risk assessment model for patients with thyroid disease by disease type will be further established. 


\section{Conclusions}

The high concentration of iodine and manganese are risk factors of thyroid disease. In addition to the low concentration of cobalt, nickel, copper, zinc, selenium, and antimony, calcium in the serum of female patients with thyroid disease and iron in the serum of male patients with thyroid disease play a very important role. Random forest model based on element risk assessment of thyroid disease is helpful for the diagnosis and treatment of thyroid disease.

\section{Abbreviations}

ICP-MS(inductively coupled plasma mass spectrometry) \FNAB (fine needle aspiration biopsy) $ه \mathrm{FT} 3$ (free Triiodothyronine), FT4 (free thyroxine), T3 (Triiodothyronine), T4 (Thyroxine), TSH (thyroid stimulating hormone), TgAbs (Thyroglobulin antibodies), TPOAb (thyroxine peroxidase), Ca (calcium), Fe(iron), Zn(zinc), I(iodine), Li(lithium) , V(vanadium),Cr(chromium), Mn(manganese), Co (cobalt), Ni (nickel), $\mathrm{Cu}$ (copper), Ga (gallium), As(arsenic), Se (selenium), Sr (strontium), Mo(molybdenum), Pd(palladium), Cd (cadmium), Sn (tin), Sb (antimony), Te (tellurium), Ba (barium), Ce (cerium), Pt (platinum), $\mathrm{Hg}$ (mercury), $\mathrm{TI}$ (thallium), $\mathrm{Pb}$ (lead), Bi (bismuth), Ge (germanium), In (indium), Tb (terbium), Rh (rhodium). USI (universal salt iodization), MUI (Median Urinary lodine), ID-I (type I deiodinase), IDD (iodine deficiency) $\mathbb{Z}$ glutathione peroxidase (GPx), Thyrotropin releasing hormone (TRH).

\section{Declarations}

\section{Ethics approval and consent to participate}

The study protocol was performed following the guidelines of the Declaration of Helsinki Principles and approved by the Institutional Review Board of Tongren Hospital (approval number 2019-0907). All participants gave informed consent before blood sampling

\section{Consent for publication}

Not applicable.

\section{Availability of data and materials}

The datasets used and/or analyzed during the current study are available from the corresponding author on reasonable request.

\section{Competing interests:}

The authors declare that they have no conflict of interest

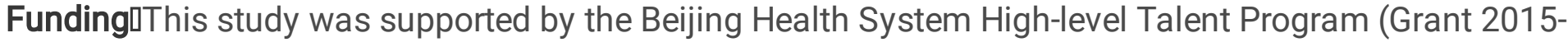
3-014).The funder had no role in the study design; the collection, analysis, and interpretation of the data; 
or in the writing of the manuscript.

Authors' contributions: $X Y L$ and J B designed the experiments. J B collected clinical samples. J B, HS L, $\mathrm{C} X$ and $\mathrm{KW} Z$ performed the experiments and analyzed data. $\mathrm{XY} L$ and $\mathrm{J} B$ interpreted the results. The manuscript was drafted by $\mathrm{J} B$ and edited by $\mathrm{C} X$ and $X Y L$. All authors read and approved the final manuscript.

Acknowledgments: The authors are grateful to the staff of Bioyong Technology Co. Ltd. for their help and instrumental support also, the authors are thankful to PhD Chan Xiong for his valuable comments and help.

\section{References}

[1] DeLellis RA, Lloyd RV, Heitz PU. World Health Organization Classification of Tumours Pathology \& Genetics. Tumours of Endocrine Organs. 2004:151-155

Article within a journal

[2] Ahn HS, Kim HJ, Welch HG. Korea's thyroid-cancer "epidemic"-screening and overdiagnosis. N Engl J Med. 2014; 371(19):1765-1767. doi:10.1056/NEJMp1409841

\section{Complete book}

[3] Kharchenko VP, Kotlyarov PM, Mogutov MS et al. Ultrasound diagnostics of thyroid diseases. 2010; Article within a journal by DOI

[4] Chen W, Zheng R, Baade PD, et al. Cancer statistics in China, 2015. CA Cancer J Clin. 2016;66(2):115132. doi:10.3322/caac. 21338

Article within a journal by DOI

[5] Navas-Carrillo D, Rodriguez JM, Montoro-García S, Orenes-Piñero E. High-resolution proteomics and metabolomics in thyroid cancer: Deciphering novel biomarkers. Crit Rev Clin Lab Sci. 2017;54(7-8):446457. doi:10.1080/10408363.2017.1394266

Article within a journal by DOI

[6] Martínez-Aguilar J, Clifton-Bligh R, Molloy MP. Proteomics of thyroid tumours provides new insights into their molecular composition and changes associated with malignancy. Sci Rep. 2016;6:23660. Published 2016 Mar 30. doi:10.1038/srep23660

Article within a journal by DOI 
[7] Haraguchi H. Metallomics as integrated biometal science. J. Anal. At. Spectrom. 2004;19:5-14 doi:10.1039/b308213j

Article within a journal by DOI

[8] Szpunar J. Metallomics: a new frontier in analytical chemistry. Anal Bioanal Chem. 2004;378(1):54-56. doi:10.1007/s00216-003-2333-z

Article within a journal by DOI

[9] Kim JY, Kim KR. Dietary iodine intake and urinary iodine excretion in patients with thyroid diseases. Yonsei Med J. 2000;41(1):22-28. doi:10.3349/ymj.2000.41.1.22

Article within a journal by DOI

[10] Kucharzewski M, Braziewicz J, Majewska U, Góźdź S. Concentration of selenium in the whole blood and the thyroid tissue of patients with various thyroid diseases. Biol Trace Elem Res. 2002;88(1):25-30. doi:10.1385/BTER:88:1:25

Article within a journal by DOI

[11] Weng W, Dong M, Zhan J, et al. A PRISMA-compliant systematic review and meta-analysis of the relationship between thyroid disease and different levels of iodine intake in mainland China [published correction appears in Medicine (Baltimore). 2018 Jan;97(1):e9593]. Medicine (Baltimore).

2017;96(25):e7279. doi:10.1097/MD.0000000000007279

Article within a journal

[12] Santos JE. Thyroid cancer consequences of iodine deficiency-a persisting worldwide problem: characteristics in different populations, portugal and south Africa. S Afr J Surg. 2017;55(3):69

Article within a journal by DOI

[13] WHO. (2007). Assessment of the iodine deficiency disorders and monitoring their elimination. WHO, Geneva, 1-107. https://doi.org/ISBN 9789241595827

Article within a journal by DOI

[14] König F, Andersson M, Hotz K, Aeberli I, Zimmermann MB. Ten repeat collections for urinary iodine from spot samples or 24-hour samples are needed to reliably estimate individual iodine status in women. J Nutr. 2011;141(11):2049-2054. doi:10.3945/jn.111.144071

Article within a journal 
[15] Zhang, J. Y. and S. M. Li, et al. Changes of the spectrum on thyroid disease after the ten-year implementation of universal salt iodization in Guangxi Zhuang Autonomous Region. Zhonghua Liuxingbingxue Zazhi 2013, 34(10): 970-974囚

Article within a journal

[16] Liu YQ, Zhang SQ, Chen WQ, Chen LL, Zheng RS. Trend of incidence and mortality on thyroid cancer in China during 2003 - 2007. Zhonghua Liu Xing Bing Xue Za Zhi. 2012;33(10):1044-1048

Article within a journal by DOI

[17] Camargo R, Knobel M, Medeiros-Neto G. lodine nutrition: more is better?. Arq Bras Endocrinol Metabol. 2007;51(4):639-640. doi:10.1590/s0004-27302007000400021

Article within a journal by DOI

[18] Rasmussen LB, Schomburg L, Köhrle J, et al. Selenium status, thyroid volume, and multiple nodule formation in an area with mild iodine deficiency. Eur J Endocrinol. 2011;164(4):585-590.

doi:10.1530/EJE-10-1026

Article within a journal by DOI

[19] Duntas LH. Selenium and the thyroid: a close-knit connection. J Clin Endocrinol Metab. 2010;95(12):5180-5188. doi:10.1210/jc.2010-0191

Article within a journal by DOI

[20] Köhrle J, Gärtner R. Selenium and thyroid. Best Pract Res Clin Endocrinol Metab. 2009;23(6):815827. doi:10.1016/j.beem.2009.08.002

Article within a journal by DOI

[21] Przybylik-Mazurek E, Zagrodzki P, Kuźniarz-Rymarz S, Hubalewska-Dydejczyk A. Thyroid disordersassessments of trace elements, clinical, and laboratory parameters. Biol Trace Elem Res. 2011;141(13):65-75. doi:10.1007/s12011-010-8719-9

Article within a journal by DOI

[22] Stazi AV, Trinti B. Selenium status and over-expression of interleukin-15 in celiac disease and autoimmune thyroid diseases. Ann Ist Super Sanita. 2010;46(4):389-399. doi:10.4415/ANN_10_04_06

Article within a journal by DOI

[23] Mazokopakis EE, Papadakis JA, Papadomanolaki MG, et al. Effects of 12 months treatment with Lselenomethionine on serum anti-TPO Levels in Patients with Hashimoto's thyroiditis. Thyroid. 
2007;17(7):609-612. doi:10.1089/thy.2007.0040

Article within a journal by DOI

[24] Turker O, Kumanlioglu K, Karapolat I, Dogan I. Selenium treatment in autoimmune thyroiditis: 9month follow-up with variable doses. J Endocrinol. 2006;190(1):151-156. doi:10.1677/joe.1.06661

Article within a journal by DOI

[25] Gärtner R, Gasnier BC, Dietrich JW, Krebs B, Angstwurm MW. Selenium supplementation in patients with autoimmune thyroiditis decreases thyroid peroxidase antibodies concentrations. J Clin Endocrinol Metab. 2002;87(4):1687-1691. doi:10.1210/jcem.87.4.8421

Article within a journal by DOI

[26] Giray B, Arnaud J, Sayek I, Favier A, Hincal F. Trace elements status in multinodular goiter. J Trace Elem Med Biol. 2010;24(2):106-110. doi:10.1016/j.jtemb.2009.11.003

Article within a journal by DOI

[27] Erdamar H, Cimen B, Gülcemal H, Saraymen R, Yerer B, Demirci H. Increased lipid peroxidation and impaired enzymatic antioxidant defense mechanism in thyroid tissue with multinodular goiter and papillary carcinoma. Clin Biochem. 2010;43(7-8):650-654. doi:10.1016/j.clinbiochem.2010.02.005

Article within a journal by DOI

[28] Keshteli AH, Hashemipour M, Siavash M, Amini M. Selenium deficiency as a possible contributor of goiter in schoolchildren of Isfahan, Iran. Biol Trace Elem Res. 2009;129(1-3):70-77. doi:10.1007/s12011008-8296-3

Article within a journal by DOI

[29] Soldin OP, Aschner M. Effects of manganese on thyroid hormone homeostasis: potential links. Neurotoxicology. 2007;28(5):951-956. doi:10.1016/j.neuro.2007.05.003

Article within a journal by DOI

[30] Rajinikanth J, Paul MJ, Abraham DT, Ben Selvan CK, Nair A. Surgical audit of inadvertent parathyroidectomy during total thyroidectomy: incidence, risk factors, and outcome. Medscape J Med, 2010;79(s1), A23-A23. doi:10.1111/j.1445-2197.2009.04916_23.x

Article within a journal by DOI

[31] Del Rio P, Montana Montana C, Cozzani F, et al. Is there a correlation between thyroiditis and thyroid cancer?. Endocrine. 2019;66(3):538-541. doi:10.1007/s12020-019-01935-8 
[32] Clinckspoor I, Verlinden L, Overbergh L, et al. 1,25-dihydroxyvitamin D3 and a superagonistic analog in combination with paclitaxel or suberoylanilide hydroxamic acid have potent antiproliferative effects on anaplastic thyroid cancer. J Steroid Biochem Mol Biol. 2011;124(1-2):1-9.

doi:10.1016/j.jsbmb.2010.12.008

Article within a journal by DOI

[33] Dabbaghmanesh MH, Sadegholvaad A, Ejtehadi F, Ranjbar-Omrani G. The role of iron deficiency in persistent goiter. Arch Iran Med. 2008;11(2):157-161. doi:08112/AIM.007

Article within a journal by DOI

[34] Kazi TG, Kandhro GA, Afridi HI, et al. Interaction of copper with iron, iodine, and thyroid hormone status in goitrous patients. Biol Trace Elem Res. 2010;134(3):265-279. doi:10.1007/s12011-009-8478-7

Article within a journal by DOI

[35] Goswami A, Rosenberg IN. Purification and partial characterization of iodothyronine 5'-deiodinase from rat liver microsomes. Biochem Biophys Res Commun. 1990;173(1):6-12. doi:10.1016/s0006$291 \times(05) 81013-7$

Article within a journal by DOI

[36] Zimmermann MB. The influence of iron status on iodine utilization and thyroid function. Annu Rev Nutr. 2006;26:367-389. doi:10.1146/annurev.nutr.26.061505.111236

Article within a journal by DOI

[37] Andersson M, Thankachan P, Muthayya S, et al. Dual fortification of salt with iodine and iron: a randomized, double-blind, controlled trial of micronized ferric pyrophosphate and encapsulated ferrous fumarate in southern India. Am J Clin Nutr. 2008;88(5):1378-1387. doi:10.3945/ajcn.2008.26149

Article within a journal by DOI

[38] Wegmüller R, Camara F, Zimmermann MB, Adou P, Hurrell RF. Salt dual-fortified with iodine and micronized ground ferric pyrophosphate affects iron status but not hemoglobin in children in Cote d'Ivoire. J Nutr. 2006;136(7):1814-1820. doi:10.1093/jn/136.7.1814

Article within a journal

[39] Soares E, Oliveira-Esquerre K, Aguiar A, Botelho GL, Kiperstok A. Development of a model to identify combined use in residential water end use events. 2018:1951-1956 
Article within a journal

[40]Robert Nisbet, John Elder, Gary Miner. Chapter 11 - Classification[J]. handbook of statistical analysis \& data mining applications, 2009:235-258.

\section{Tables}

Table 1 Instrumental settings

\begin{tabular}{|ll|}
\hline parameters & Set value \\
\hline RF power & $1400 \mathrm{~W}$ \\
\hline Atomization chamber temperature & $3^{\circ} \mathrm{C}$ \\
\hline Atomizing gas & $0.980 \mathrm{~L} \cdot \mathrm{min}^{-1}$ \\
\hline Auxiliary gas & $1.00 \mathrm{~L} \cdot \mathrm{min}^{-1}$ \\
\hline Cooling gas & $14.00 \mathrm{~L} \cdot \mathrm{min}^{-1}$ \\
\hline He flow & $0.72 \mathrm{~mL} \cdot \mathrm{min}^{-1}$ \\
\hline Sampling depth & $3 \mathrm{~mm}$ \\
\hline
\end{tabular}

Table 2 The contents of elements with a significant difference between healthy people and patients with thyroid disease

\begin{tabular}{|c|c|c|c|c|c|c|c|c|}
\hline \multirow[t]{2}{*}{ Elements } & \multicolumn{3}{|c|}{ Healthy $(n=50)$} & \multicolumn{3}{|c|}{ Thyroid $(n=200)$} & \multirow[t]{2}{*}{$\mathrm{p}$} & \multirow[t]{2}{*}{ Unit } \\
\hline & Mean & $\mathrm{SD}$ & RSD & Mean & SD & RSD & & \\
\hline I & 85.9 & 18.5 & $22 \%$ & 168 & 19.6 & $12 \%$ & $<0.01$ & $\mu \mathrm{g} / \mathrm{L}$ \\
\hline $\mathrm{Ca}$ & 101 & 6.9 & $7 \%$ & 97.8 & 17.1 & $18 \%$ & $<0.05$ & $\mathrm{mg} / \mathrm{L}$ \\
\hline $\mathrm{Fe}$ & 2.0 & 0.6 & $31 \%$ & 1.7 & 0.5 & $32 \%$ & $<0.01$ & $\mathrm{mg} / \mathrm{L}$ \\
\hline $\mathrm{Ni}$ & 4.8 & 0.7 & $16 \%$ & 2.6 & 0.7 & $28 \%$ & $<0.01$ & $\mu \mathrm{g} / \mathrm{L}$ \\
\hline $\mathrm{Cu}$ & 1162 & 359 & $31 \%$ & 765 & 220 & $29 \%$ & $<0.01$ & $\mu \mathrm{g} / \mathrm{L}$ \\
\hline $\mathrm{Zn}$ & 945 & 143 & $15 \%$ & 593 & 136 & $23 \%$ & $<0.01$ & $\mu \mathrm{g} / \mathrm{L}$ \\
\hline $\mathrm{Se}$ & 109 & 19.9 & $18 \%$ & 84.9 & 21.2 & $25 \%$ & $<0.01$ & $\mu \mathrm{g} / \mathrm{L}$ \\
\hline $\mathrm{Sb}$ & 3.4 & 0.6 & $17 \%$ & 2.0 & 0.6 & $33 \%$ & $<0.01$ & $\mu \mathrm{g} / \mathrm{L}$ \\
\hline
\end{tabular}

Table 3 Content of elements by sex in healthy people and patients with thyroid diseases 


\begin{tabular}{|c|c|c|c|c|c|c|c|c|c|}
\hline \multirow[t]{3}{*}{ Elements } & \multicolumn{4}{|c|}{ Healthy } & \multicolumn{4}{|c|}{ Thyroid } & \multirow[t]{3}{*}{ Unit } \\
\hline & \multicolumn{2}{|c|}{$\operatorname{Man}(n=19)$} & \multicolumn{2}{|c|}{ Women $(n=31)$} & \multicolumn{2}{|c|}{$\operatorname{Man}(n=39)$} & \multicolumn{2}{|c|}{ Women $(n=161)$} & \\
\hline & Mean & SD & Mean & SD & Mean & SD & Mean & SD & \\
\hline I & 79.3 & 11.0 & 89.9 & 20.9 & 173 & 20.6 & 166 & 19.1 & $\mu g / L$ \\
\hline $\mathrm{Ca}$ & 101 & 7.66 & 102 & 6.3 & 99.7 & 20.8 & 97.3 & 16.1 & $\mathrm{mg} / \mathrm{L}$ \\
\hline $\mathrm{Cr}$ & 1.92 & 0.92 & 1.8 & 0.8 & 3.7 & 3.8 & 3.5 & 1.9 & $\mu g / L$ \\
\hline $\mathrm{Mn}$ & 1.44 & 0.32 & 1.3 & 0.3 & 1.9 & 1.0 & 1.6 & 1.1 & $\mu g / L$ \\
\hline $\mathrm{Fe}$ & 2.54 & 0.45 & 1.7 & 0.5 & 2.0 & 0.7 & 1.7 & 0.5 & $\mathrm{mg} / \mathrm{L}$ \\
\hline Co & 0.43 & 0.08 & 0.6 & 0.3 & 0.04 & 0.1 & 0.2 & 0.3 & $\mu g / L$ \\
\hline $\mathrm{Ni}$ & 4.62 & 0.70 & 4.9 & 0.8 & 2.6 & 0.9 & 2.6 & 0.7 & $\mu g / L$ \\
\hline $\mathrm{Cu}$ & 997 & 192 & 1263 & 398 & 728 & 211 & 775 & 221 & $\mu g / L$ \\
\hline $\mathrm{Zn}$ & 980 & 161 & 924 & 126 & 637 & 174 & 583 & 123 & $\mu g / L$ \\
\hline As & 1.38 & 2.46 & 1.0 & 0.8 & 0.8 & 0.8 & 1.0 & 3.0 & $\mu g / L$ \\
\hline $\mathrm{Se}$ & 116 & 23.4 & 105 & 16.0 & 89.1 & 25.8 & 83.9 & 19.8 & $\mu g / L$ \\
\hline Mo & 1.12 & 0.37 & 1.1 & 0.4 & 10.0 & 14.0 & 8.0 & 13.2 & $\mu \mathrm{g} / \mathrm{L}$ \\
\hline $\mathrm{Pd}$ & 1.59 & 1.05 & 5.5 & 7.0 & 0.5 & 0.3 & 0.6 & 0.3 & $\mu \mathrm{g} / \mathrm{L}$ \\
\hline $\mathrm{Cd}$ & 0.13 & 0.09 & 0.1 & 0.1 & 7.4 & 11.8 & 6.1 & 11.1 & $\mu \mathrm{g} / \mathrm{L}$ \\
\hline $\mathrm{Sb}$ & 3.38 & 0.66 & 3.4 & 0.5 & 2.1 & 0.6 & 1.9 & 0.7 & $\mu \mathrm{g} / \mathrm{L}$ \\
\hline TI & 0.02 & 0.01 & 0.02 & 0.01 & 0.02 & 0.01 & 0.02 & 0.01 & $\mu \mathrm{g} / \mathrm{L}$ \\
\hline $\mathrm{Pb}$ & 2.34 & 2.72 & 0.05 & 0.04 & 0.49 & 0.77 & 0.45 & 1.33 & $\mu \mathrm{g} / \mathrm{L}$ \\
\hline
\end{tabular}

Table 4 Significant difference of element content between female healthy people and female patients with thyroid disease 


\begin{tabular}{|c|c|c|c|c|c|c|c|c|}
\hline \multirow{2}{*}{$\begin{array}{l}\text { Women } \\
\text { Elements }\end{array}$} & \multicolumn{3}{|c|}{ Healthy $(n=31)$} & \multicolumn{3}{|c|}{ Thyroid $(n=161)$} & \multirow[t]{2}{*}{$p$} & \multirow[t]{2}{*}{ Unit } \\
\hline & Mean & SD & RSD & Mean & SD & RSD & & \\
\hline I & 89.9 & 20.9 & $23 \%$ & 166 & 19.1 & $11 \%$ & $<0.01$ & $\mu \mathrm{g} / \mathrm{L}$ \\
\hline $\mathrm{Ca}$ & 102 & 6.3 & $6 \%$ & 97.3 & 16.1 & $17 \%$ & $<0.05$ & $\mathrm{mg} / \mathrm{L}$ \\
\hline $\mathrm{Cr}$ & 1.8 & 0.8 & $46 \%$ & 3.5 & 1.9 & $54 \%$ & $<0.01$ & $\mu \mathrm{g} / \mathrm{L}$ \\
\hline $\mathrm{Mn}$ & 1.3 & 0.3 & $22 \%$ & 1.6 & 1.1 & $67 \%$ & $<0.05$ & $\mu \mathrm{g} / \mathrm{L}$ \\
\hline Co & 0.6 & 0.3 & $45 \%$ & 0.2 & 0.3 & $179 \%$ & $<0.01$ & $\mu \mathrm{g} / \mathrm{L}$ \\
\hline $\mathrm{Ni}$ & 4.9 & 0.8 & $15 \%$ & 2.6 & 0.7 & $27 \%$ & $<0.01$ & $\mu \mathrm{g} / \mathrm{L}$ \\
\hline $\mathrm{Cu}$ & 1263 & 398 & $32 \%$ & 775 & 221 & $29 \%$ & $<0.01$ & $\mu \mathrm{g} / \mathrm{L}$ \\
\hline $\mathrm{Zn}$ & 924 & 126 & $14 \%$ & 583 & 123 & $21 \%$ & $<0.01$ & $\mu \mathrm{g} / \mathrm{L}$ \\
\hline $\mathrm{Se}$ & 105 & 16.0 & $15 \%$ & 83.9 & 19.8 & $24 \%$ & $<0.01$ & $\mu \mathrm{g} / \mathrm{L}$ \\
\hline $\mathrm{Sb}$ & 3.4 & 0.5 & $15 \%$ & 1.9 & 0.7 & $35 \%$ & $<0.01$ & $\mu \mathrm{g} / \mathrm{L}$ \\
\hline
\end{tabular}

Table 5 element contents with a significant difference between male healthy people and male patients with thyroid disease

\begin{tabular}{|c|c|c|c|c|c|c|c|c|}
\hline Men & Health & $(n=19$ & & Thyroi & $(n=39$ & & $p$ & Unit \\
\hline Elements & Mean & SD & RSD & Mean & SD & RSD & & \\
\hline I & 79.3 & 11.0 & $14 \%$ & 173 & 20.6 & $12 \%$ & $<0.01$ & $\mu \mathrm{g} / \mathrm{L}$ \\
\hline $\mathrm{Mn}$ & 1.44 & 0.32 & $22 \%$ & 1.9 & 1.0 & $55 \%$ & $<0.05$ & $\mu \mathrm{g} / \mathrm{L}$ \\
\hline $\mathrm{Fe}$ & 2.54 & 0.45 & $18 \%$ & 2.0 & 0.7 & $36 \%$ & $<0.01$ & $\mathrm{mg} / \mathrm{L}$ \\
\hline $\mathrm{Ni}$ & 4.62 & 0.70 & $15 \%$ & 2.6 & 0.9 & $32 \%$ & $<0.01$ & $\mu g / L$ \\
\hline $\mathrm{Cu}$ & 997 & 192 & $19 \%$ & 728 & 211 & $29 \%$ & $<0.01$ & $\mu g / L$ \\
\hline $\mathrm{Zn}$ & 980 & 161 & $16 \%$ & 637 & 174 & $27 \%$ & $<0.01$ & $\mu g / L$ \\
\hline $\mathrm{Se}$ & 116 & 23.4 & $20 \%$ & 89.1 & 25.8 & $29 \%$ & $<0.01$ & $\mu g / L$ \\
\hline $\mathrm{Sb}$ & 3.38 & 0.66 & $20 \%$ & 2.1 & 0.6 & $26 \%$ & $<0.01$ & $\mu \mathrm{g} / \mathrm{L}$ \\
\hline
\end{tabular}

Table 6 Correlation between content of $\mathrm{Fe}, \mathrm{Zn}, \mathrm{Cr}, \mathrm{Mn}, \mathrm{Ca}$, Se, and trace elements in blood serum in the different groups 


\begin{tabular}{|lcccc|}
\hline Correlation between parameters & \multicolumn{2}{l}{ Control grounp $(\mathrm{n}=50)$} & \multicolumn{2}{l|}{ Thyroid disease group $(\mathrm{n}=183)$} \\
\cline { 2 - 6 } & $r_{\text {Pearson }}$ & $p$ & $r_{\text {Pearson }}$ & $p$ \\
\hline Fe-Zn & 0.2563 & $p>0.05$ & 0.5523 & $p<0.05$ \\
\hline Cr-Mn & 0.1333 & $p>0.05$ & 0.5528 & $p<0.05$ \\
\hline Ca-Zn & 0.3608 & $p<0.05$ & 0.7158 & $p<0.05$ \\
\hline Ca-Se & 0.5466 & $p<0.05$ & 0.5699 & $p<0.05$ \\
\hline Ca-Fe & 0.0483 & $p>0.05$ & 0.6371 & $p<0.05$ \\
\hline Zn-Se & 0.0691 & $p>0.05$ & 0.5420 & $p<0.05$ \\
\hline
\end{tabular}

Table 7 Random forest effect evaluation

\begin{tabular}{|lll|}
\hline group & Specificity & sensitivity \\
\hline Health and thyroid nodules & $97.20 \pm 3.29 \%$ & $99.6 \pm 1.36 \%$ \\
\hline Health and thyroid cancer & $97.73 \pm 3.43 \%$ & $99.67 \pm 1.72 \%$ \\
\hline Health and thyroid postoperation & $98.80 \pm 2.56 \%$ & $99.87 \pm 0.93 \%$ \\
\hline Health and Goiter & $98.80 \pm 2.56 \%$ & $95.33 \pm 5.92 \%$ \\
\hline
\end{tabular}

Figures 

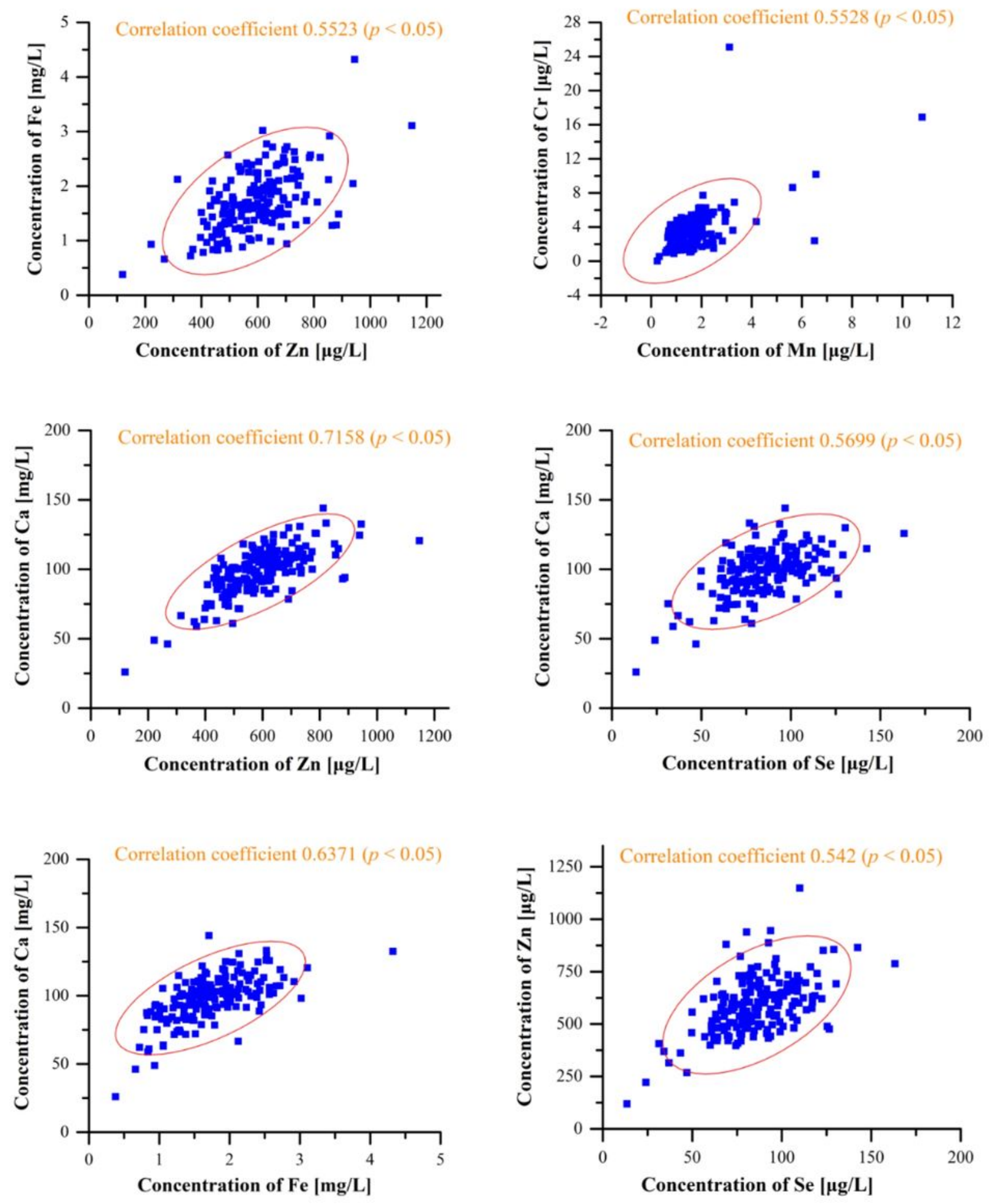

\section{Figure 1}

There are related trace elements in patients with thyroid disease $(n=183), a)$ Fe-Zn; b) Cr-Mn $₫ c \rrbracket C a-Z n \rrbracket d \rrbracket$ Ca-Se $₫ e \otimes C a-F e \otimes f \bigotimes Z n-S e$ 

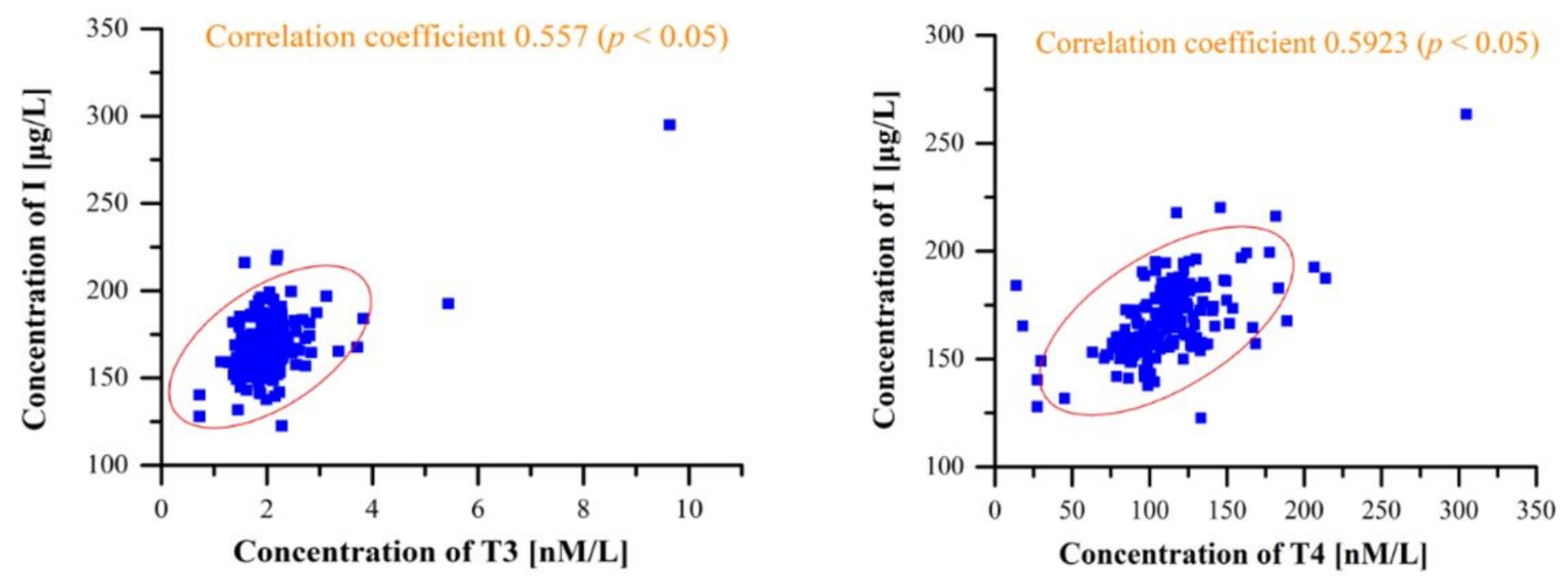

Figure 2

The correlation between the content of trace elements and the value of thyroid function in patients with thyroid disease $(n=174) \llbracket a \rrbracket l-T 3 \bigotimes b$ bl-T4
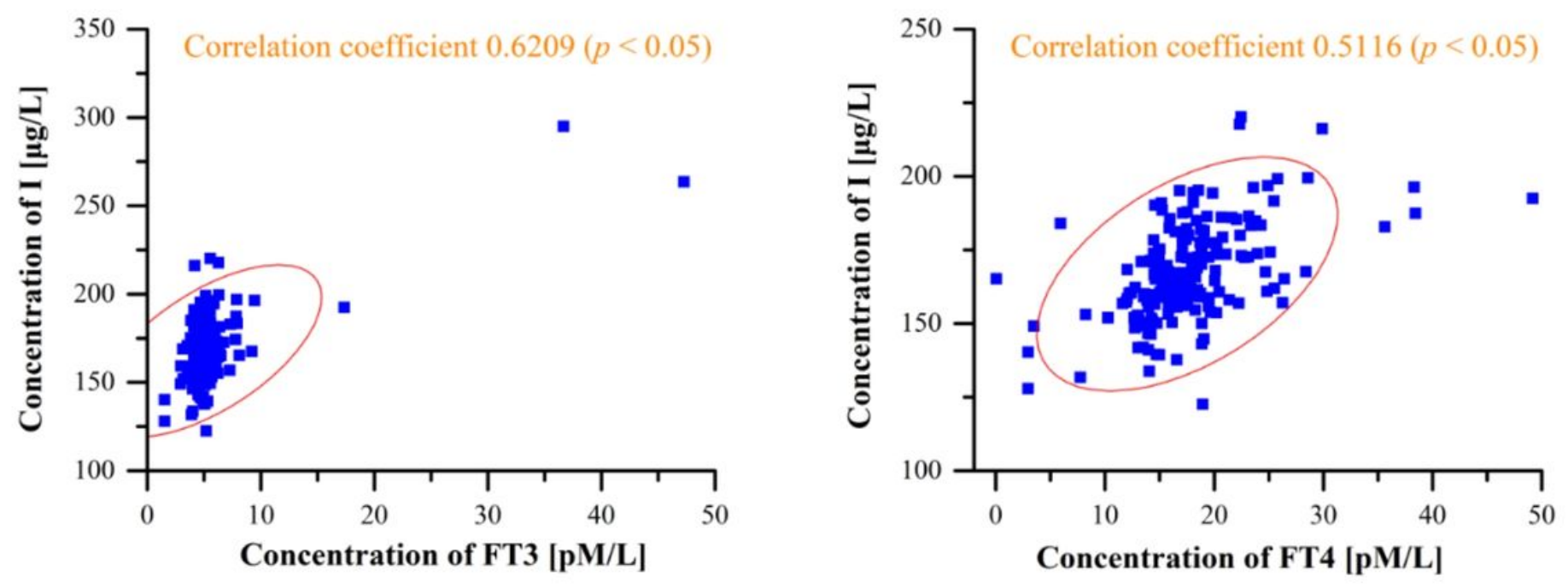

Figure 3

The correlation between the content of trace elements and the value of thyroid function in patients with

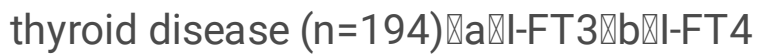




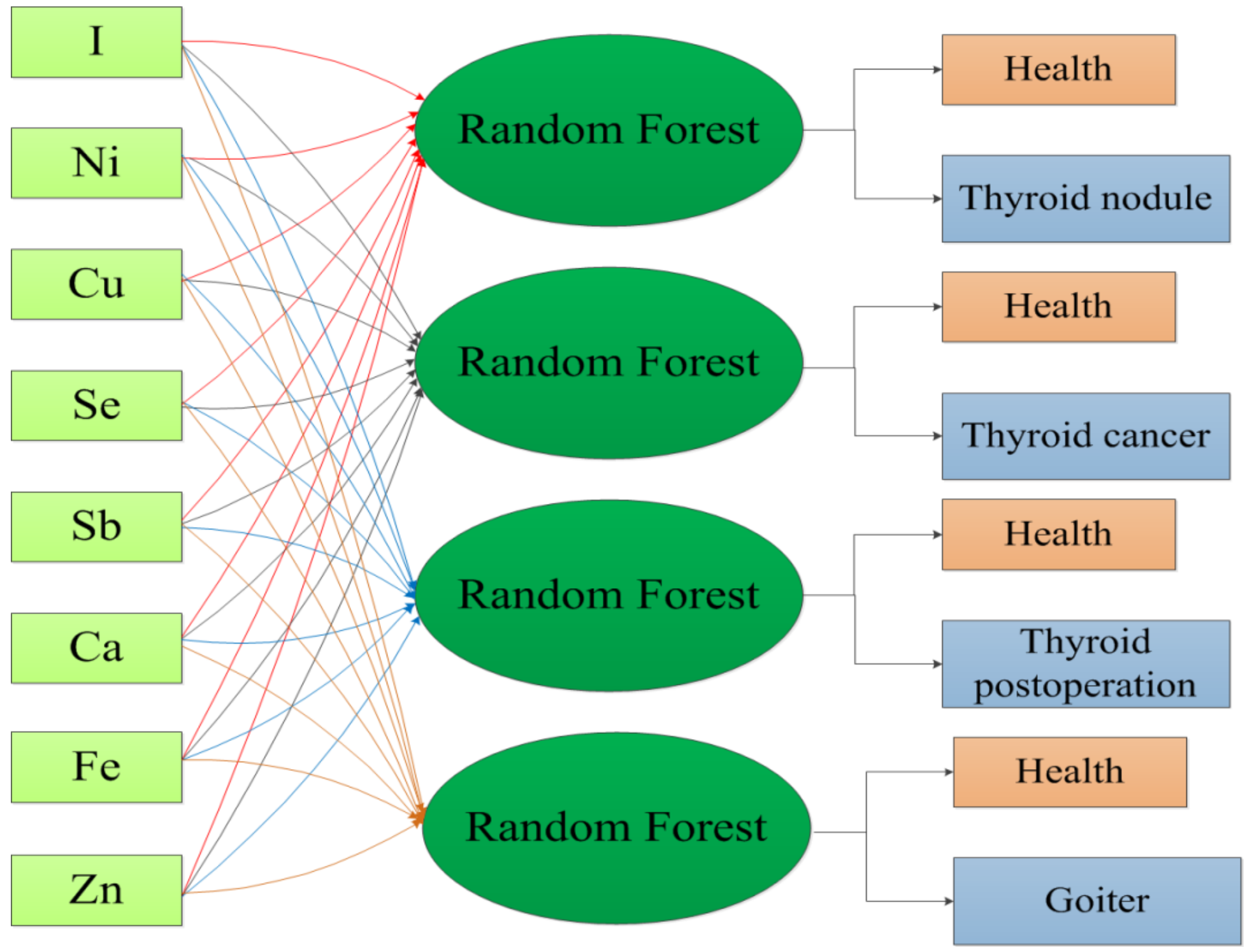

Figure 4

Schematic diagram of random forest model 